\title{
Immunohistochemical analysis of cannabinoid receptor 1 expression in steatotic rat livers
}

\author{
KRZYSZTOF ZDUNIAK ${ }^{1}$, PIOTR ZIÓŁKOWSKI ${ }^{1}$, PONTUS REGNELL ${ }^{2}$, PETRA TOLLET-EGNELL ${ }^{3}$, \\ LINA ÅKESSON $^{4}$ and MARTIN E. COOPER ${ }^{2}$ \\ ${ }^{1}$ Department of Pathology, Wrocław Medical University, Wrocław PL-50-368, Poland; ${ }^{2}$ AstraZeneca R\&D Mölndal, \\ Mölndal SE-431 83; ${ }^{3}$ Department of Molecular Medicine and Surgery, The Karolinska Institute, \\ Karolinska University Hospital, Stockholm SE-171 76; ${ }^{4}$ Diabetes and Celiac Unit, \\ Faculty of Medicine, Lund University, Malmö 205 02, Sweden
}

Received January 31, 2015; Accepted November 2, 2015

DOI: $10.3892 /$ etm.2016.3036

\begin{abstract}
The primary aim of the present study was to determine the expression levels of cannabinoid receptor type 1 (CB1) in steatotic rat livers. The secondary aim was to clarify whether steatosis and inflammation are more marked in areas with increased CB1 overexpression. For ethical and economic reasons, the present study investigated tissue from archived liver blocks, which were obtained from 38 rats that had been euthanized during the course of previous research at the Karolinska Institute of the Karolinska University Hospital (Stockholm, Sweden) and Lund University (Malmö, Sweden). Liver tissue fixed in formalin and embedded in paraffin was used that had been sourced from 36 male Sprague Dawley rats (age, 7 weeks) and 2 rats (age, 180 days) lacking normal leptin receptors. The rat liver tissue was stained with antibodies against CB1 and counterstained with hematoxylin. The expression of CB1 and the number of cells overexpressing CB1 were determined. Steatosis was scored according to the Dixon scoring system. CB1 overexpression and steatosis were detected in hepatocytes from all 38 livers sampled. The expression of CB1 was more marked in hepatocytes localized next to portal triads. Near the central veins, the expression was significantly weaker. Steatosis was more marked in areas of increased CB1 overexpression. Lymphocyte infiltration was more commonly observed in areas of increased CB1 overexpression. Therefore, the present results indicate that $\mathrm{CB} 1$ receptors are overexpressed in areas with steatosis, and indicate that CB1 in hepatocytes contributes to the formation of steatosis in rats, even prior to its progression to steatohepatitis. These results are consistent
\end{abstract}

Correspondence to: Mr. Krzysztof Zduniak, Department of Pathology, Wrocław Medical University, ul K. Marcinkowskiego 1, Wrocław PL-50-368, Poland

E-mail: kzdun91@gmail.com

Key words: cannabinoid receptor 1, immunohistochemistry, rats, fatty liver with publications reporting that $\mathrm{CB} 1$ in hepatocytes increases lipogenesis and contributes to inflammation.

\section{Introduction}

Cannabinoid receptor type 1 (CB1) has been found to be involved in intrahepatocy te lipid accumulation $(1,2)$. CB1 overexpression in hepatocytes has been hypothesized to contribute to the formation of hepatic steatosis and inflammation (3-5). Steatogenic agents, such as ethanol, and a high-fat diet can upregulate the activity of CB1 via the increased synthesis of endocannabinoids, 2-arachidonoylglycerol, and anandamide (4). CB1 receptor activation upregulates the lipogenic transcription factor, sterol regulatory element-binding protein 1c and its target enzymes, acetyl-CoA carboxylase-1 and fatty acid synthase. Furthermore, CB1 receptor activation downregulates carnitine palmitoyltransferase-1, which is the rate-limiting enzyme associated with fatty acid oxidation. Therefore, CB1 activation increases fatty acid synthesis and decreases fatty acid oxidation, which results in a fatty liver (4). Previously published images of hepatic CB1 overexpression in human diseases have predominantly been obtained from patients suffering from serious liver diseases, such as severe non-alcoholic fatty liver disease found in morbid obesity (6), stage IV primary biliary cirrhosis (7) and hepatitis $C$ (8). However, it remains unclear whether CB1 overexpression additionally contributes to milder, more common liver diseases, such as fatty liver disease without or with mild inflammation.

The primary aim of the present exploratory study was to determine the extent of CB1 expression in steatotic rat livers. A secondary aim was to clarify whether steatosis and inflammation are more extensive in areas of increased CB1 overexpression.

\section{Materials and methods}

Animals. A total of 36 male Sprague Dawley rats obtained from the Karolinska Institute of the Karolinska University Hospital (Stockholm, Sweden) had free access to regular rodent chow (R36, Lactamin, Kimstad, Sweden) and 
water. For $14(n=12)$ or 21 days $(n=5)$, the water for 17 of the rats contained $10 \%$ fructose; whereas the remaining 19 rats received fructose-free water. Duration of fructose treatment was selected in order to induce hepatic steatosis and various severities of insulin resistance. Nine rats additionally received one injection of insulin $(1 \mathrm{mU} / \mathrm{g}$ actrapid; Novo Nordisk, Bagsvaerd, Denmark) at 40 min prior to euthanasia. All institutional (Karolinska Institute) and Swedish national guidelines for the care and use of laboratory animals were followed. The rats were sacrificed at 7 weeks of age in the course of other research. All animal experiments were approved by the regional Ethics Committee on Animal Research (North Stockholm, Sweden). Following the experiments, formalin-fixed and paraffin-embedded liver slices from the rats were obtained. The Karolinska Institute provided slices from the middle of the right median lobe for $\mathrm{CB} 1$ staining, along with a list of durations of fructose exposure.

Two male rats, aged 180 days that were homozygous for leptin receptor gene mutations (9) were bred at the Lund University. The rats lacked the C-terminal end of the leptin receptor, which completely inhibited leptin-receptor binding and function. These rats received Lantmännen R3 breeding chow for rat and mouse (Lantmännen, Stockholm, Sweden). The rats were hyperleptinemic and obese. All institutional (Lund University) and Swedish national guidelines for the care and use of laboratory animals were followed. The research was approved by the Malmö/Lund Ethics Committee for Animal Experiment. The rats were euthanized at 180 days of age in the course of other research. Spare formalin-fixed and paraffin-embedded liver blocks were provided for CB1 staining.

Immunohistochemical staining. Formalin-fixed and paraffin-embedded liver blocks were sectioned at $4 \mu \mathrm{m}$, deparaffinized in two changes of xylene (9 min each) and rehydrated in alcohols (96, 80 and $70 \%$ for 1 min each). Sections were then washed twice in distilled water and placed in $0.01 \mathrm{M}$ sodium citrate ( $\mathrm{pH}$ 6.0) in a microwave oven for $9 \mathrm{~min}$ for heat-induced epitope retrieval. Following two 5-min washes in distilled water, specimens were incubated for 10 min with Peroxidase Blocking Reagent (Dako, Glostrup Denmark) and rinsed twice with phosphate-buffered saline (PBS), for 5 min each time. Incubation with Protein Block Reagent was performed for $15 \mathrm{~min}$, then specimens were incubated with rabbit primary antibodies (CB1; 10006590; Cayman Chemical Company, Ann Arbor, MI, USA) at a concentration of 1:50 for $1 \mathrm{~h}$ at room temperature, and then rinsed twice with PBS, for 5 min each time. Subsequently, the goat secondary antibody (Dako EnVision+System-HRP anti-rabbit; K4065; Dako) was applied and incubated for $30 \mathrm{~min}$ at room temperature. Following rinsing twice with PBS for 5 min each time, 3,3'-diaminobenzidine (Dako) was applied to the samples at the original dilution, and after the next rinsing (twice for $5 \mathrm{~min}$ each rinse) in distilled water, the slides were counterstained with hematoxylin. Following washing in tap water for $10 \mathrm{~min}$, the slides were covered with cover slips. Negative controls were created by omitting the first antibodies.

Grading and scoring. Steatosis was graded according to the system proposed by Dixon et al (10). The Dixon system
Table I. Scoring of the liver tissue samples.

\begin{tabular}{lcc}
\hline & \multicolumn{2}{c}{ Livers (n) } \\
\cline { 2 - 3 } Score & CB1 & Dixon \\
\hline 1 & 0 & 5 \\
2 & 3 & 4 \\
3 & 8 & 15 \\
4 & 11 & 14 \\
5 & 9 & - \\
6 & 7 & - \\
7 & 0 & -
\end{tabular}

CB1, cannabinoid receptor 1 .

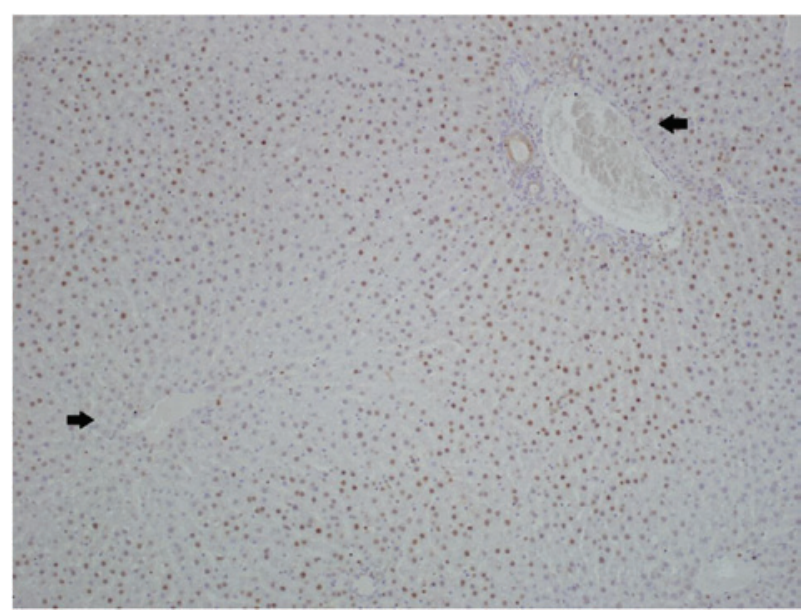

Figure 1. Cannabinoid receptor 1 (CB1) expression topography. Relatively weak intracellular presentation of CB1 receptor was observed in hepatocytes near the central vein, which is represented by the arrow on the left, compared with the hepatocytes localized near the portal triad, which are represented by the arrow on the right (magnification, $\mathrm{x} 200$ ).

quantifies steatosis, whereas the present system measured CB1. Furthermore, in order to determine the extent of inflammatory activity, the Bedossa scoring system was used (11), where the activity score (A; from 0-4) was the sum of hepatocyte ballooning (0-2) and lobular inflammation (0-2). A0 indicates no activity. The immunoreactivity of CB1 was evaluated using two parameters: i) Intensity of staining; and ii) number of stained cells. Briefly, score 1 was assigned to weak staining, 2 to moderate staining and 3 to strong. To report the number of CB1-stained cells, a system based on the aforementioned Dixon system was used, as follows: Score 1, 0-5\% CB1-stained cells; score 2, 5-25\% CB1-stained cells; score 3, 25-75\% CB1-stained cells; and score 4, >75\% CB1-stained cells. The total CB1 score was calculated by adding the two scores together, and therefore ranged between 1 and 7 points. Two pathologists independently evaluated all histological slides using a BX51 light microscope (Olympus Corporation, Tokyo, Japan). Images were captured using a DP10 digital camera (Olympus Corporation) at magnifications of $\times 100$ and $\times 200$. 


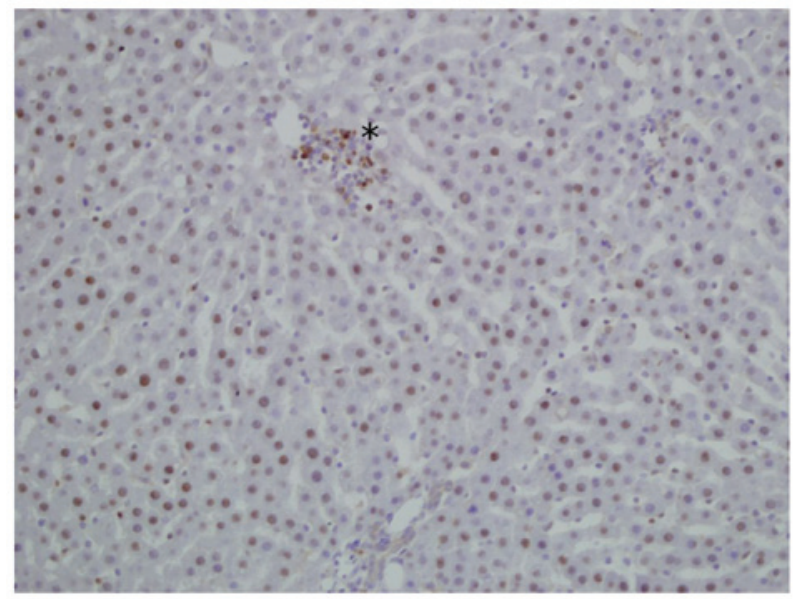

Figure 2. Cannabinoid receptor 1 (CB1) expression in lymphocytes. Intracellular presentation of CB1 receptor in hepatocytes and in a small lymphocytic infiltration which is represented by the asterisk (magnification, $\mathrm{x} 200)$.

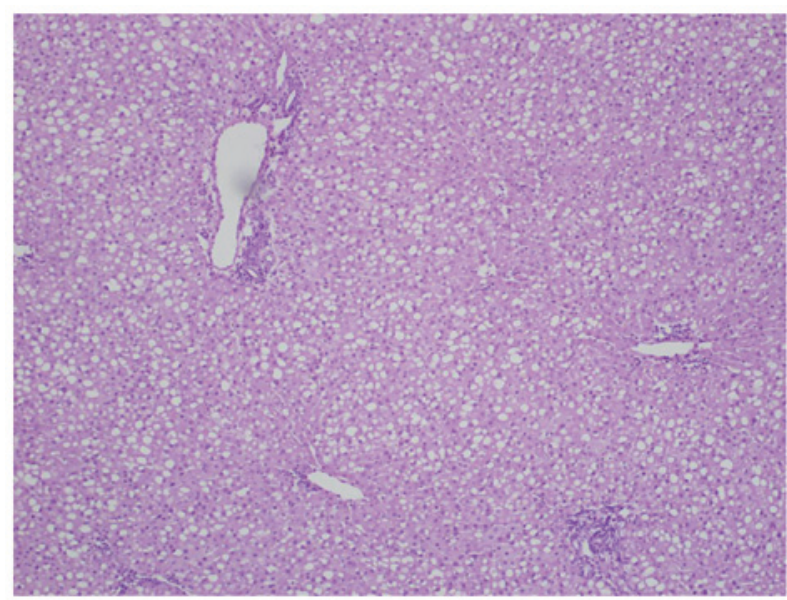

Figure 3. Dixon's stage 4 steatosis of the liver with a diffuse pattern (magnification, $\mathrm{x} 100$ ).

\section{Results}

All cases exhibited CB1 expression, which coexisted with steatosis. Positive staining for CB1 was observed in a large proportion of the hepatocytes in each case. The total score of positive staining (i.e. the score for intensity of staining plus the score for the number of stained cells) is shown in Table I. Two out of the seven cases that scored 6 were from the rats lacking normal leptin receptors.

The expression of CB1 was more marked in hepatocytes located next to portal triads and significantly reduced in hepatocytes near to the central veins (Fig. 1). Similar results were obtained for steatosis, which was less marked in hepatocytes near to the central veins. Therefore, increased CB1 expression and steatosis coexisted to a considerable extent. In addition, immunohistochemical staining for CB1 receptor was also performed in certain smaller groups of intralobular lymphocytic inflammatory infiltrations in the liver samples (Fig. 2). Inflammatory activity represented by lymphocytic infiltrations was slightly more common in samples that exhibited increased CB1 expression; however, the inflamma- tory activity did not exceed $\mathrm{A} 0$ grade of the Bedossa scoring system.

The liver samples from obese rats lacking normal leptin receptors scored a total CB1 score of 6 and exhibited macrovesicular grade 4 steatosis, whereas the livers from Sprague Dawley rats exhibited a reduced CB1 score and/or less severe steatosis.

Details of steatosis. The stage of steatosis was determined based on the Dixon system (10). Respective Dixon scores are presented in Table I. In 20 cases the steatosis was microvesicular, whereas in 18 cases the steatosis was macrovesicular. The two rats lacking normal leptin receptors exhibited stage 4 macrovesicular steatosis. Hematoxylin-eosin staining revealed stage 4 hepatic steatosis with a diffuse pattern (Fig. 3).

\section{Discussion}

Previously published images of CB1 expression have predominantly been captured from cases with severe liver diseases, including severe non-alcoholic fatty liver disease found in morbid obesity (6), stage IV primary biliary cirrhosis (7) and hepatitis C (8). Liu et al (6) have previously captured immunohistochemistry images of human livers stained with CB1 antibodies. In these figures, the nuances of the hepatocytes in the liver with no obvious pathology appear identical to the negative control, which had not been stained with CB1. Therefore, the immunohistochemistry images captured in the present study and those attained by Liu et al (6) suggested that the visible CB1 expression levels exhibited in the present study constitute overexpression. The present study used similar CB1 antibodies to those that generated the aforementioned images (6-8). The present results indicated that CB1 was overexpressed in the hepatocytes of rats with steatosis with mild or without any inflammatory activity in two strains of rats (Figs. 1 and 2). In these images, steatosis was most marked in areas exhibiting the overexpression of CB1. This is consistent with previous studies, which have reported that CB1 contributes to the formation of steatosis $(4,12)$. In addition, lymphocyte infiltrations were more commonly observed in areas where CB1 was highly overexpressed, which is consistent with studies that have suggested that CB1 contributes to inflammation (12) and plays a role in inflammatory cells (13). In particular, a CB1 antagonist, rimonabant, has been demonstrated to suppress the lipopolysaccharide-induced production of the pro-inflammatory interleukins (IL), IL-6 and IL-8 in human macrophages (13).

The livers extracted from the 2 rats lacking normal leptin receptors had a total $\mathrm{CB} 1$ score of 6 , and macrovesicular grade 4 steatosis. By contrast, the livers from the 36 Sprague Dawley rats exhibited a reduced CB1 score and/or less severe steatosis. These results may have been due to the increased age of the rats lacking normal leptin receptors, which allowed more time for disease progression. High CB1 activity has previously been associated with obesity (14) and hepatic steatosis.

The present results suggested that hepatic CB1 contributes to steatosis in rats, even prior to its progression to steatohepatitis. Although the present results are preliminary and require further confirmation, it is to be expected that a hepatic CB1 antagonist that is actively transported into hepatocytes may 
exert a protective effect against steatosis and inflammatory complications. Previous studies have suggested that a hepatic CB1 antagonist may be designed that would be actively transported into hepatocytes $(12,15)$. For example, a glucokinase activator has been optimized for active liver uptake, leading to a $>50$-fold increase in free concentration in the liver compared with that in the pancreas (15). Furthermore, a hepatoselective CB1 antagonist may be capable of ameliorating steatosis, insulin resistance, dyslipidemia and inflammatory liver disease (12). The present results suggest that a hepatoselective CB1 antagonist could ameliorate steatosis and steatohepatitis in rodents, even prior to the occurrence of serious complications. Centrally acting CB1 antagonists are known to produce notable psychiatric side effects in certain patients (16); however a hepatoselective CB1 antagonist may have the potential to be effective with negligible psychiatric side effects (12).

The present study investigated tissue from spare, archived liver blocks, which were obtained from 38 rats that had been euthanized during the course of previous research at the Karolinska Institute of the Karolinska University Hospital and Lund University in order to keep the number of animals used for research to a minimum. Therefore, among the limitations of this study is the lack of individual data for the food and fructose intake levels of the rats per day, in addition to the final body weight of the rats. However, these variables do not appear to be necessary for the present investigation. In the livers of 7-week-old Sprague-Dawley rats in a previous study (17), the triglyceride content did not differ significantly between males and females, which supports the generalization of the present findings to female rats. In addition, to the best of our knowledge, no previous results have suggested that the hepatic molecular pathways associated with steatosis differ markedly between males and females. Another limitation of the present study is that the descriptive statics are not supplemented with inferential statistics. The scoring system used assigned identical scores to tissues with steatosis ranging from 26 to $75 \%$; however, these tissues may not have had identical disease severity. In future studies, actual percentages, rather than scores, should be recorded as a basis for calculating correlations between CB1 upregulation, steatosis and inflammation

On the basis of the present histological findings and the results of previous studies on CB1 overexpression in more serious inflammatory liver diseases, we have come to the conclusion that it is likely that CB1 overexpression contributes to increased steatosis and complications across several stages of the disease, including the early stages.

\section{Acknowledgements}

The authors thank Ms Linda Faxius from the Diabetes and Celiac Unit (Faculty of Medicine, Lund University) for her assistance in obtaining tissue from rats lacking normal leptin receptors.

\section{References}

1. De Gottardi A, Spahr L, Ravier-Dall'Antonia F and Hadengue A: Cannabinoid receptor 1 and 2 agonists increase lipid accumulation in hepatocytes. Liver Int 30: 1482-1489, 2010.

2. Pai WY, Hsu CC, Lai CY, Chang TZ, Tsai YL and Her GM: Cannabinoid receptor 1 promotes hepatic lipid accumulation and lipotoxicity through the induction of SREBP-1c expression in zebrafish. Transgenic Res 22: 823-838, 2013.

3. Auguet T, Berlanga A, Guiu-Jurado E, Terra X, Martinez S, Aguilar C, Filiu E, Alibalic A, Sabench F, Hernández M, et al: Endocannabinoid receptors gene expression in morbidly obese women with nonalcoholic fatty liver disease. Biomed Res Int 2014: 502542, 2014.

4. Purohit V, Rapaka R and Shurtleff D: Role of cannabinoids in the development of fatty liver (Steatosis). AAPS J 12: 233-237, 2010.

5. Osei-Hyiaman D, DePetrillo M, Pacher P, Liu J, Radaeva S, Bátkai S, Harvey-White J, Mackie K, Offertáler L, Wang L and Kunos G: Endocannabinoid activation at hepatic CB1 receptors stimulates fatty acid synthesis and contributes to diet-induced obesity. J Clin Invest 115: 1298-1305, 2005.

6. Liu J, Zhou L, Xiong K, Godlewski G, Mukhopadhyay B, Tam J, Yin S, Gao P, Shan X, Pickel J, et al: Hepatic cannabinoid receptor-1 mediates diet-induced insulin resistance via inhibition of insulin signaling and clearance in mice. Gastroenterology 142: 1218-1228.e1, 2012.

7. Floreani A, Lazzari R, Macchi V, Porzionato A, Variola A, Colavito D, Leon A, Guido M, Baldo V, De Caro R and Bergasa NV: Hepatic expression of endocannabinoid receptors and their novel polymorphisms in primary biliary cirrhosis. J Gastroenterol 45: 68-76, 2010.

8. van der Poorten D, Shahidi M, Tay E, Sesha J, Tran K, McLeod D, Milliken JS, Ho V, Hebbard LW, Douglas MW and George J: Hepatitis C virus induces the cannabinoid receptor 1. PLoS One 5: e12841, 2010.

9. Moralejo DH, Hansen CT, Treuting P, Hessner MJ, Fuller JM, Van Yserloo B, Jensen R, Osborne W, Kwitek AE and Lernmark A: Differential effects of leptin receptor mutation on male and female BBDR Gimap5-/Gimap5-spontaneously diabetic rats. Physiol Genomics 41: 9-20, 2010.

10. Dixon JB, Bhathal PS, Hughes NR and O'Brien PE: Nonalcoholic fatty liver disease: Improvement in liver histological analysis with weight loss. Hepatology 39: 1647-1654, 2004.

11. Bedossa P, Poitou C, Veyrie N, Bouillot JL, Basdevant A, Paradis V, Tordjman J and Clement K: Histopathological algorithm and scoring system for evaluation of liver lesions in morbidly obese patients. Hepatology 56: 1751-1759, 2012.

12. Cooper ME and Regnell SE: The hepatic cannabinoid 1 receptor as a modulator of hepatic energy state and food intake. Br J Clin Pharmacol 77: 21-30, 2014.

13. Kaplan BL: The role of CB1 in immune modulation by cannabinoids. Pharmacol Ther 137: 365-374, 2013.

14. Matias I, Gonthier MP, Orlando P, Martiadis V, De Petrocellis L, Cervino C, Petrosino S, Hoareau L, Festy F, Pasquali R, et al: Regulation, function, and dysregulation of endocannabinoids in models of adipose and beta-pancreatic cells and in obesity and hyperglycemia. J Clin Endocrinol Metab 91: 3171-3180, 2006.

15. PfefferkornJA,Guzman-PerezA,LitchfieldJ,AielloR,TreadwayJL, Pettersen J,Minich ML,Filipski KJ, Jones CS, Tu M, et al:Discovery of (S)-6-(3-cyclopentyl-2-(4-(trifluoromethyl)-1H-imidazol-1-yl) propanamido) nicotinic acid as a hepatoselective glucokinase activator clinical candidate for treating type 2 diabetes mellitus. J Med Chem 55: 1318-1333, 2012.

16. Christensen R, Kristensen PK, Bartels EM, Bliddal H and Astrup A: Efficacy and safety of the weight-loss drug rimonabant: A meta-analysis of randomised trials. Lancet 370: 1706-1713, 2007.

17. Gustavsson C, Yassin K, Wahlström E, Cheung L, Lindberg J, Brismar K, Ostenson CG, Norstedt G and Tollet-Egnell P: Sex-different hepatic glycogen content and glucose output in rats. BMC Biochem 11: 38, 2010. 